\title{
Dynamic Queue and TCP New Reno based Multipath Load Balancing Congestion Control Scheme for Mobile Ad Hoc Networks (MANET)
}

\author{
Purushesh Shukla \\ M.Tech. Scholar \\ VNS Institute Of Technology \\ Bhopal, (M.P.), India
}

\author{
Tushar Kanti \\ Assistant Professor \\ VNS Institute Of Technology \\ Bhopal, (M.P.), India
}

\begin{abstract}
In Effective load balancing has been a difficult task in Mobile Ad hoc Networks (MANET) due to their frequent change in topology. Multipath routing are providing the alternative path that is identified from source to destination using intermediate nodes that have better communication and processing capabilities to take part in the mobile routing and efficiently participate in the routing process but only multipath are not sufficient for handle congestion in network because it only provides the alternate path not provides the effective load balancing in network. This paper presents a new congestion control scheme based on multipath routing for enhanced load balancing in MANET. This scheme enhanced load balancing from dynamic queue length method that improves the store and forwarding capacity of nodes in network and to maintained queue size data rate of sender are controlled by that the TCP New Reno scheme. The combination of these two approaches provides better performance and congestion control according to current network traffic levels and nodes' processing loads. The proposed scheme performance are evaluate AOMDV performance and observed that the performance of proposed scheme showing the zero packets loss in terms of queue length and also minimizes the congestion. The performance is also measured on the basis of performance metrics like routing overhead, delay and the whole simulation is done in ns-2 simulator.
\end{abstract}

\section{Keywords}

Multipath, MANET, Congestion, Load balancing, Queue length, TCP Reno.

\section{INTRODUCTION}

The wireless network can be characteristics into a local area wireless network, ad hoc network and Satellite wireless network. Mobile Ad hoc Network (MANET) [1] is one kind of wireless network that does not need a support of centralized infrastructure to be set up for connection establishment and data delivery. The advantages of ad hoc network are ease of deployment than wired networks. Each node acts as a host and a router that moves arbitrarily and communicates via multiple wireless links. The example of MANET is shown in figure 1, where the nodes are independent and free to communicate with each other without any supervision. The typical areas of mobile ad hoc network applications include battlefields, emergency search rescue sites and data acquisition in remote areas. A mobile ad hoc network is characterized by a topology where nodes enter and leave the network dynamically. The networks have limited channel bandwidth and require multiple hops to exchange information with any other node in the network. The packets delivering over wireless links also suffer from radio intervention of neighboring mobile nodes. The topological change due to mobility and limited battery power capacity of nodes have to be deal with without causing long link break periods.

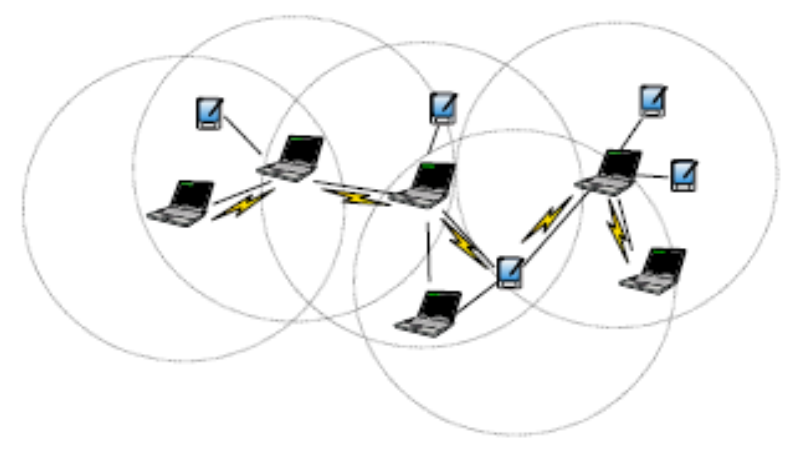

Fig1: Mobile Ad hoc Network

The excessive load on the nodes is cause packet dropping due to the buffer overflow. This leads to enhance the end to end delay and affects the packet delivery ratio of MANET protocol. Load balancing [2] is a solution to avoid congestion in the network by proving alternative path. If the load is balanced then it will provide efficient use of the network and reduce packet delay and improve packet delivery ratio. The existing protocols provide us with multiple paths but fail to avoid congestion and provide load balancing. AOMDV [3] provides multiple routes to a destination, selects a single route with low hop count and discards higher hop count routes. These routes might be utilized as alternate routes in case of link failure. To provide effective utilization of the network the load must be transferred to alternative paths. A factor Queue Length gives information about the number of packets present in the buffer of a nodes. With the help of Queue length we determine whether a network is congested or not. If Queue Length is more required then alternate paths are taken in order to provide Load Balancing in the network. To avoid congestion in the network the broadcast of route request packets is avoided. Congestion could also be avoided by selecting routes on the basis of hop count and Queue Length of each node.

Now, TCP New Reno [4] is more capable when multiple packets losses occur because of congestion. New Reno correspond to go through fast retransmit when multiple duplicate packets received, but it does not come out from fast recovery phase until all exceptional data was not acknowledged. It mean that in New Reno, partial ACK do not take TCP out of fast recovery but they are treated as an indicator that the packet in the sequence space has been lost, and should be retransmitted. Therefore, when multiple packets are lost from a single window of data, at this time New Reno can improve without retransmission time out. The retransmitting rate is one packet loss per round trip time until 
all of the lost packets from that window have been transmitted. It exist in fast recovery till all the data is injected into network, and still waiting for an acknowledgement that fast recovery was initiated. The faster retransmission capability is rapidly inform to sender through Acknowledgement (ACK) to slow transmission rate.

\section{PREVIOUS WORK ACCOMPLISHED}

In this section we present the research that has done in this field.

In this paper [5] a technique for Multipath Load Balancing and Rate Based Congestion Control (MLBRBCC) is presented. In this technique, sender node delivers the data packet to the destination node through the intermediate nodes. The receiving of the data packet, the bandwidth utilization percentage and queue length are estimated at each intermediate node along the destination. On the bases of these values congestion status and estimated rate are calculated and transmitted towards the destination. By checking the updated values from the intermediate nodes, the destination node determines the estimated rate and it is transmitted as a feedback to the sender. The sender performs rate control based on the estimated rate in the feedback packet.

In this paper [6], proposed a approach when the average load of an available link increases beyond a defined threshold value and the available bandwidth capacity and residual battery energy decreases below a particular defined threshold value then traffic is distributed over fail-safe multiple routes to reduce the traffic load on a congested link. The simulation results, are show that this approach achieves better throughput and packet delivery ratio with reduced delay for constant bit rate (CBR) traffic when compared with QMRB ( a protocol using QoS mobile routing backbones).

In this paper [7], proposed protocol proficiently provides QoS multipath load balanced routing capabilities and improved robustness with low routing overhead in network. The selection of multi-paths avoid the repetitive selection of alternate routes and avoid minimum less efficient paths (i.e. congested, low energy paths) to participate in routing. This load balancing scheme works by selecting multiple weighted paths and follows a bandwidth reservation mechanism to improve the connection throughput significantly. The weight computation process considers not only the necessary parameters but also the service classes of the network.

In this paper [8], the proposed method for avoid congestion in MANET environment using bandwidth estimation technique. This scheme uses acknowledgement time intervals for bandwidth estimation in TCP flow, in this scheme it monitor the time spacing between received acknowledgements (ACKs) at the sender node and find out the available bandwidth of the connection between sender to destination, if available bandwidth is less than the actual data size so decrease the data size according to available bandwidth and avoid congestion, and same time other sender node try to communicate any destination through same available path which is used by previously senders so new sender node and all other previously communicated sender node sends data according to available bandwidth of the intermediate nodes so we increase the network performance and provides congestion free communication.

In this paper [9], proposed a node-disjoint multipath routing approach based on AODV protocol. The proposed route discovery method identifies all the available node-disjoint routes using a single flooding of a RREQ message. This scheme reduces the routing overhead that has occurred due to route discovery and maintenance processes thus increasing the network capacity. This method is suitable for low and moderate mobility of nodes in networks. Also, three different route maintenance methods are proposed and implemented to show the performance of their route discovery method. To reduce the initial delay, source node can send data as soon as it gets the selected (Primary) route. Due to multiple routes stored in routing table backup routes are always available for continuous data transmission when the selected route is broken.

In this paper [10] the problem of Optimized Link State Routing (OLSR)'s for instance the routing loop problems can be solved, for, this protocol still have the self-interference problem. There is an intricate interdependence between MAC layers and routing layer, it deal with congestion control for multi-flow traffic in wireless mobile ad-hoc networks (MANET). For simulations, it used ns-3 open source network simulator considering Optimized Link State Routing (OLSR) routing protocol. The MANET proposed performance considering in random waypoint mobility model for different number of nodes and different area sizes, by sending multiflow traffic in the network. We found that coupling congestion control mechanisms between multi-flows have problems in some cases.

\section{PROBLEM STATEMENT}

Congestion in a network may occur at interval time when the incoming traffic is larger than the capacity of the network. The problem of congestion are not possible to remove completely in network but possible to detect it and minimize it. This network congestion can severely increase delay and packet loss and cut the network throughput. Congestion control refers to techniques that can keep away from congestion before it happens or recovery after it happens. The problem of congestion in MANET has occurred because of following reasons:-

- Limited bandwidth constraint.

- Limited buffer capacity of intermediate nodes.

- Absence of Multipath routing procedure.

- Efficient load balancing means that distribute load properly.

- Route changes due to node mobility as well as the inherently unreliable medium result in unsteady packet delivery delays and packet losses.

\section{PROPOSED WORK}

In our proposal we minimize data drop through acknowledgement delay estimation base rate selection as well as dynamic queue base drop minimization, very first we set initial parameter of MANET and after that we select best possible multiple path from source to destination, and then we send data, in that case we apply TCP New Reno updated technique and calculate acknowledgement delay difference and set new data size for transmission the data but if incoming rate is faster than the outgoing rate then we apply dynamic queue scheme for minimization data drop from the network TCP New Reno are alert the sender for slow your transmission. The main advantage of this research is to combine the two approaches for congestion control that provides the outstanding results.

Step 1: Mobile node N;

Step 2: Set Radio Range 550 meters

Step 3: Sender S subset of N

Step 4: Receiver R subset of $\mathrm{N}$

Step 5: Set routing AOMDV

Step 6: Ack is acknowledgment 
Step 7: Sender B_RREQ to next-> neighbour;

While (next- $>$ neighbour $<=550$ \&\& next- $>$ neighbour! = Receiver)

Accept route requested packet;

Forward B_RREQ to next neighbour;

If (next->neighbour $==$ Receiver)

$\{$ Create route table;

Check more than one route table;

Send (R_ACK to sender node through more than one path)

Else $\{$ Check route request limit time;

If (RRLT $>=$ Route search time)

\{Exit $0 ;\}$

Step 8: Sender send (data, S, R)

Step 9: if (intermediate $\mathrm{Q}==$ Full)

\{

Q incr +1 ;

Enqueued-data in Q;

Else \{

Data in Q;

\}

Step 10: Receiver send (Ack, R, S)

Step 11:Ack- Delay()

$$
\text { Delay }=\mathrm{Ack}_{\mathrm{n}}-\mathrm{Ack}_{\mathrm{n}-1}
$$

Step 12: set new-data-Size =Previous data size/Delay;

Step 13: Stop Simulation;

\section{SIMULATION ENVIRONMENT \& RESULTS}

The Network Simulator (Version 2), widely known as NS2, is simply an event driven simulation tool that has proved useful in studying the dynamic nature of communication networks. Simulation of wired, wireless as well as ad hoc network functions and protocols like UDP, TCP etc. can be done using NS2.

In general, NS2 provides users with a way of specifying such network protocols and simulating their corresponding behaviors. Due to its flexibility and modular nature, NS2 has gained constant popularity in the networking research community since its birth in 1989. Ever since, several revolutions and revisions have marked the growing maturity of the tool, thanks to substantial contributions from the players in the field. Among these are the University of California and Cornell University who developed the REAL network simulator, 1 the foundation which NS is based on. Since 1995 the Defense Advanced Research Projects Agency (DARPA) supported development of NS through the Virtual Inter Network Test bed (VINT) project [11]. Currently the National Science Foundation (NSF) has joined the ride in development. Last but not the least, the group of researchers and developers in the community are constantly working to keep NS2 strong and versatile.

\subsection{Performance Metrics}

We are taking the following parameters for case study i.e. shown in Table 1.
Table 1. Simulation parameter

\begin{tabular}{|l|l|}
\hline Number of nodes & 50 \\
\hline Dimension of simulated area & $800 \times 800$ \\
\hline Routing Protocol & AOMDV \\
\hline Simulation time (seconds) & 100 \\
\hline Queue Length & 50 (dynamic if required more) \\
\hline Transport Layer & TCP (TCP New reno),UDP \\
\hline Antenna Type & Omni Antenna \\
\hline Traffic type & CBR, FTP \\
\hline Packet size (bytes) & 1000 \\
\hline Number of traffic connections & 4 TCP, 3 UDP \\
\hline Node mobility (m/s) & Random \\
\hline
\end{tabular}

\subsection{Results}

In this section we examine the results analysis on the basis of performance metrics and different drop reasons.

\subsubsection{Packet Delivery Ratio Analysis}

Packet Delivery Ratio (PDR) is the fraction of number of packets received by number of packets send in network. This graph represents the PDR analysis in case of normal AOMDV routing this is the PDR Old performance. But in proposed PDR performance the queue and TCP New Reno are minimizes the possibility of congestion in network by handle network load. In this scheme the load balancing is done through dynamic queue buffer size, it improves the store and forward capacity and by TCP New Reno the data rate are managed by ACK to sender. Here we clearly visualized that the PDR in case of Normal AOMDV and proposed AOMDV are nearly equal up to time 65 seconds but after that the performance of proposed AOMDV are showing the better performance than normal AOMDV.

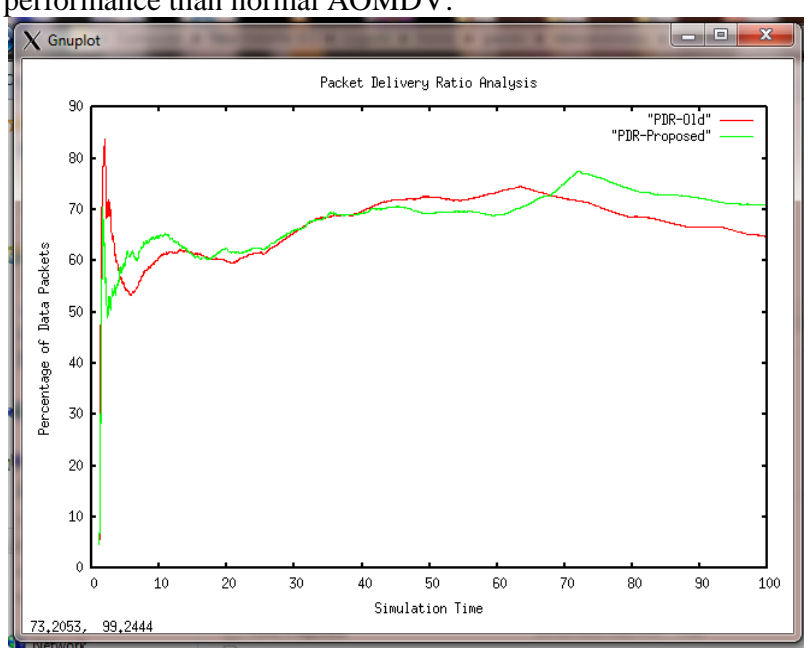

Fig 2: PDR Analysis

\subsubsection{Routing Load analysis}

Routing Load analysis is the routing packets or control packets analysis that are deliver on network by sender for connection establishment to receiver. The receiver confirm the path through connection confirmation packets that is also establishment packet that gives the information to sender, receiver is ready for receiving data. This graph represents the 
routing load analysis in case of normal AOMDV (Old Routing) and proposed AOMDV routing. The Dynamic Queue management technique with control data rate performance with TCP New Reno is improved the routing load in case of proposed scheme. In this graph the proposed and Old performance are visualized almost equal but the exact value of routing load is given in Table 3 overall summery i.e. showing the exact improved performance of proposed scheme. This proposed scheme efficiently balance the load by that the retransmission is not needed as a result routing overhead decreases.

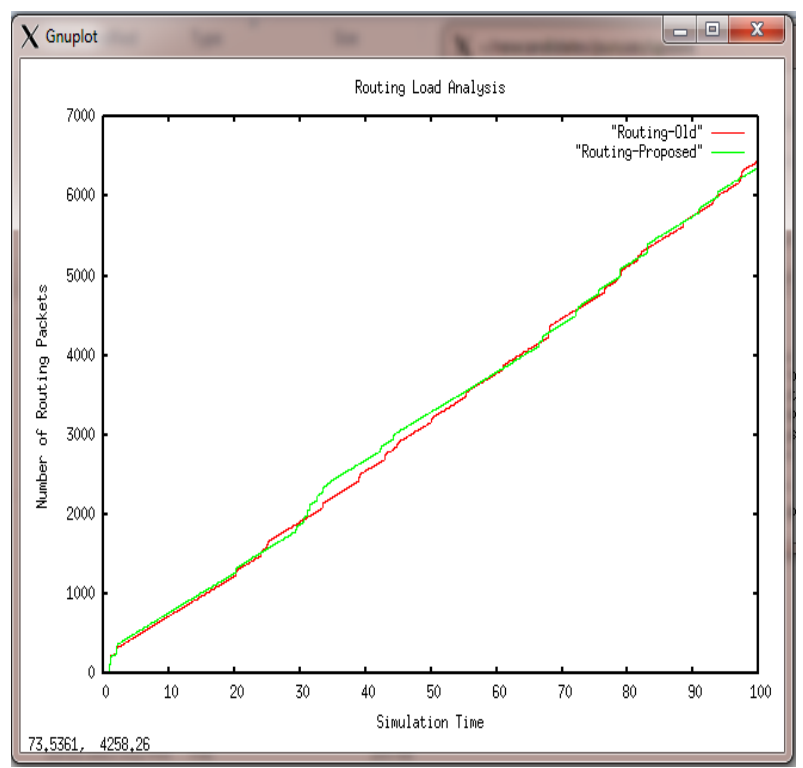

Fig 3: Routing Load Analysis

\subsubsection{Throughput Analysis}

Throughput is measured on the basis of number of packets received at destination in per unit of time. The quantity of efficient packet delivery in terms of loss through congestion in network is less represents the better throughput. The throughput in case of normal AOMDV routing is nearly equal to 100 packets/second in network till at the end of simulation but in case of proposed AOMDV routing with improvement of dynamic queue technique and bandwidth estimation through TCP New Reno are improved the performance of throughput. Here the throughput in case of proposed scheme is showing the more than 300 packets/second in time between 1 to 22 seconds in network but after that the throughput is continuously decreases from 300 and reaches to 150 packets/ seconds at time about 42 seconds but after that the throughput is maintained at 200 packets/seconds at the end of simulation. It means that the throughput in case of proposed scheme is absolutely more than double as Old scheme.

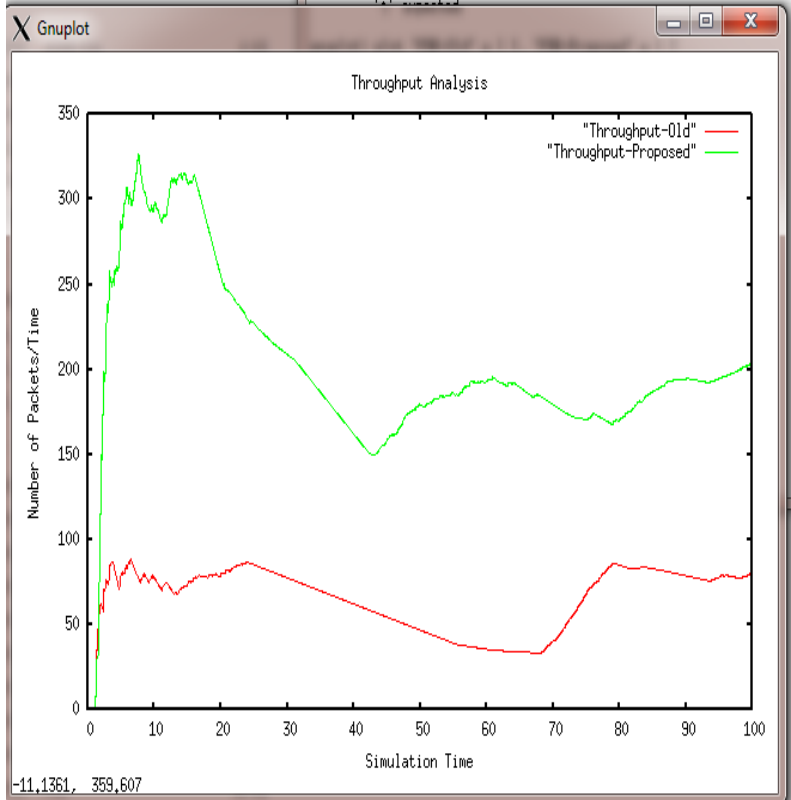

Fig. 4 Throughput Analysis

\subsubsection{Overall Performance Summary of old and} Proposed Scheme

The overall analysis in case of old scheme and proposed scheme are mentioned in Table 2 . Here we clearly observed the performance of proposed scheme is improves the routing mechanism and represents better results than old scheme.

Table 2. Performance Summary

\begin{tabular}{|l|c|l|c|l|}
\hline PARAMETERS & \multicolumn{2}{|c|}{ Old Scheme } & \multicolumn{2}{c|}{$\begin{array}{c}\text { Proposed } \\
\text { Scheme }\end{array}$} \\
\hline SEND & 11827 & & 11649 & \\
\hline RECV & 7649 & & 8246 & \\
\hline ROUTINGPKTS & 6447 & & 6372 & \\
\hline PDF & 64.67 & & 70.79 & \\
\hline NRL & 0.84 & & 0.77 & \\
\hline DROPRTS & 96 & & 51 & \\
\hline No. of dropped data & 4178 & & 3403 & \\
\hline $\begin{array}{c}\text { Actual } \\
\text { Performance }\end{array}$ & 25923 & $75.15 \%$ & 11649 & $\begin{array}{c}87.98 \\
\%\end{array}$ \\
\hline
\end{tabular}

\subsubsection{Different Packets Drop Reasons Analysis}

There are many reasons of packet dropping in network but here we consider some of them in Table 3 and observe the performance of Old scheme and proposed scheme on the basis of that parameters. Here in proposed scheme due to queue length zero (0) packets are represents and also minimizes the congestion in network. Rest of the parameters excluded ARP are showing the better performance in network. 
Table 3. Packet Drop Analysis

\begin{tabular}{|l|l|l|l|l|}
\hline Drop Reasons & \multicolumn{2}{|l|}{ Old Case } & \multicolumn{2}{l|}{ Proposed Case } \\
\hline Drop from ARP & 21 & $0.06 \%$ & 35 & $0.12 \%$ \\
\hline Drop from IFQ & 4057 & $11.76 \%$ & 0 & $0.00 \%$ \\
\hline Drop from CBK & 138 & $0.40 \%$ & 96 & $0.32 \%$ \\
\hline Drop from NRT & 80 & $0.23 \%$ & 4 & $0.01 \%$ \\
\hline $\begin{array}{l}\text { Total Drop Via } \\
\text { Congestion }\end{array}$ & 4274 & $12.39 \%$ & 3454 & $11.57 \%$ \\
\hline Total Drop & 8570 & $24.85 \%$ & 3589 & $12.02 \%$ \\
\hline
\end{tabular}

\section{CONCLUSION}

Temporary connection is maintained between the nodes in Mobile ad hoc Network (MANET) and frequent change in topology is creating the problem in routing procedure. The limited bandwidth capacity and limited node storing and forwarding capacity causes the problem of congestion in MANET. Now multipath routing handles the problem of congestion by leaving the congested route and providing the alternative path from source to destination but AOMDV does not provide the efficient load balancing. In this paper we proposed congestion control AOMDV is based on load balancing to minimize the possibility of congestion in MANET. In this technique the random queue approach and TCP New Reno approach provides better performance as compared to normal AOMDV. The simulation results illustrate that the proposed scheme is showing the zero packet dropping due to queue length in network and minimizes packet dropping because of congestion. Rest of the performance metrics also gives the better results and improves the routing capability of AOMDV protocol.

In future we will try to apply proposed approach on Ant colony optimization technique to improve the pheromones based routing approach.

\section{REFERENCES}

[1] P.Goyal, V.Parmer, R.rishi, "MANET: Vulnerabilities, Challenges, Attacks, Application", IJCEM, Vol. 11, pp. 32-37, January 2011.
[2] M. Al-Tarazi, "Load Balancing Using Multiple Paths in Mobile Ad Hoc Networks [dissertation]." Irbid: Jordan University of Science and Technology, 2009.

[3] Mahesh K. Marina and Samir R. Das, "On-Demand Multipath Distance Vector Routing in Ad Hoc Networks" in IEEE Proceedings 2001.

[4] S. Floyd, T. Henderson and A. Gurtov, "The New Reno Modification to TCP's Fast Recovery Algorithm," RFC 3782, Apr. 2004.

[5] S. Soundararajan and R.S. Bhuvaneswaran, "Multipath Load Balancing \& Rate Based Congestion Control for Mobile Ad Hoc Networks (MANET)" IEEE 2012 Second International Conference on Digital Information and Communication Technology and it's Applications (DICTAP), pp. 30-35, 2012

[6] M. Ali, B.G Stewart, A.Shahrabi, A. Vallavaraj, "Congestion adaptive multipath routing for load balancing in Mobile Ad hoc Networks", IEEE International Conference on Innovations in Information Technology (IIT), pp. 305 - 309, 18-20 March 2012.

[7] Nitin Goel, Shruti Sangwan, Ajay Jangra, "Efficient Weighted innovative Routing Protocol (EWIRP) to Balance Load in Mobile Ad Hoc Networks (MANETs)", IEEE-International Conference On Advances In Engineering, Science And Management (ICAESM 2012) , pp. 278-284, March 30, 31, 2012

[8] Ramratan Ahirwal and Ganesh Lokhande, "TCP Congestion Control through Bandwidth Estimation Mechanism in MANET", International Journal of Applied Information Systems (IJAIS) - ISSN : 22490868 Foundation of Computer Science FCS, New York, USA Volume 2- No.1, pp. 1-8, May 2012.

[9] Chhagan Lal ,V.Laxmi, M.S.Gaur "A Node-Disjoint Multipath Routing Method based on AODV protocol for MANETs" 2012 26th IEEE International Conference on Advanced Information Networking and Applications, pp399-405, 2012

[10] Makoto Ikeda, Elis Kulla, Masahiro Hiyama, Leonard Barolli, Rozeta Miho and Makoto Takizawa, "Congestion Control for Mul i-flow Traffic in Wireless Mobile Ad-hoc Networks", IEEE Sixth International Conference on Complex, Intelligent, and Software Intensive Systems, pp. 290-297, 2012.

[11] The Network Simulator - ns-2. [Online]. Available: http://www .isi.edu/nsnam/ns/ 